\title{
Paving the way toward highly efficient, high-energy potassium-ion batteries with ionic-liquid electrolytes
}

\author{
Michele Fiore ${ }^{a, b, \dagger}$, Samuel Wheeler ${ }^{a, \dagger}$, Kevin Hurlbutt ${ }^{a}$, Isaac Capone ${ }^{a}$, Jack Fawdon ${ }^{a}$, \\ Riccardo Ruffo ${ }^{b}$, Mauro Pasta ${ }^{a, \star}$ \\ a. Department of Materials, University of Oxford, Parks Road, Oxford OX1 3PH, UK \\ b. Department of Materials Science, Università di Milano-Bicocca, Via Roberto Cozzi \\ 55, 20125, Milano, Italy \\ * Correspondence: mauro.pasta@materials.ox.ac.uk
}

\begin{abstract}
Potassium-ion batteries (KIB) are a promising complementary technology to lithium-ion batteries because of the comparative abundance and affordability of potassium. Currently, the most promising KIB chemistry consists of a potassium manganese hexacyanoferrate (KMF) cathode, a Prussian blue analog, and a graphite anode $\left(723 \mathrm{Wh} \mathrm{h}^{-1}\right.$ and $359 \mathrm{Wh} \mathrm{kg}^{-1}$ at $\left.3.6 \mathrm{~V}\right)$. No electrolyte has yet been formulated that is concurrently stable at the high operating potential of $\mathrm{KMF}\left(4.02 \mathrm{~V}\right.$ vs $\left.\mathrm{K}^{+} / \mathrm{K}\right)$ and compatible with $\mathrm{K}^{+}$intercalation into graphite, currently the most critical hurdle to adoption. Here we combine a KMF cathode and a graphite anode with a KFSI in $\mathrm{Pyr}_{1,3} \mathrm{FSI}$ ionic liquid electrolyte for the first time and show unprecedented performance. We use high-throughput techniques to optimize the KMF morphology for operation in this electrolyte system, achieving $119 \mathrm{~mA} \mathrm{~h} \mathrm{~g}^{-1}$ at $4 \mathrm{~V} \mathrm{vs} \mathrm{K}^{+} / \mathrm{K}$ and a coulombic efficiency $>99.3 \%$. In the same ionic liquid electrolyte graphite shows excellent electrochemical performance and we demonstrate reversible cycling by operando XRD. These results are a significant and essential step forward towards viable potassium-ion batteries.
\end{abstract}

\section{Introduction}

While lithium-ion batteries currently dominate electrochemical energy storage globally, there are substantial concerns about the long-term availability, price, price stability, and supply-chain security and reliability of lithium and cobalt, two critical elements in lithium-ion batteries. ${ }^{1,2}$ Potassium-ion batteries, shown schematically in Figure 1, are a technology analogous to lithium-ion but with several competitive advantages. ${ }^{3}$ They replace rare and expensive lithium with abundant and inexpensive potassium. Moreover, the less dense and less expensive aluminium can be used instead of copper at the negative current collector because, unlike lithium, potassium does not alloy with aluminum. ${ }^{4}$ This avoids the issue of overdischarge which allows shipping in the discharged state. Unlike sodium-ion batteries, another promising alternative to lithiumion, they can use current graphite anodes since potassium intercalates into graphite. ${ }^{5-7}$ Graphite, with a theoretical specific capacity of $279 \mathrm{~mA} \mathrm{~h} \mathrm{~g}^{-1}$ and an operating voltage of $0.3 \mathrm{~V}$ vs $\mathrm{K}^{+} / \mathrm{K}$, is the most promising KIB anode material, but good electrochemical performance is dependent on electrolyte formulation ${ }^{8}$ and binder choice. ${ }^{7}$ The two main challenges to KIB adoption are developing energy-dense active materials for the cathode and formulating electrolytes that are electrochemically stable at high potentials whilst also being compatible with the graphite anode.

Candidate potassium-ion cathode materials include layered oxides, polyanion compounds, and Prussian blue analog (PBAs). ${ }^{3}$ Layered metal oxides typically have reasonable specific capacities but operate at low voltages and have poor cyclability. ${ }^{9}$ The best performing polyanion compounds, $\mathrm{KVPO}_{4} \mathrm{~F}$ and $\mathrm{KVOPO}_{4}$, have high operating voltages $\left(>4 \mathrm{~V}\right.$ vs. $\mathrm{K}^{+} / \mathrm{K}$ and reasonable specific capacities $\left(\approx 100 \mathrm{~mA} \mathrm{~h} \mathrm{~g}^{-1}\right)$ but contain toxic and costly vanadium. ${ }^{10,11}$

The most promising class of cathodes for KIBs are PBAs as they have high voltages, highest capacities and have potential to be low-cost materials as they are produced by an aqueous synthesis route from earth-abundant precursors. They are a family of compounds with a nanoporous, openframework crystal structure. ${ }^{12}$ Their lattice contains two electrochemically active metal centers bridged by cyanide ligands with an interstitial sites capable of accommodating an inserting alkali-metal ion. Their general formula is

$$
\mathrm{A}_{\mathrm{x}} \mathrm{M}^{\mathrm{J}}\left[\mathrm{M}^{\prime \mathrm{K}}(\mathrm{CN})_{6}\right]_{1-\mathrm{y}} \cdot \mathrm{wH}_{2} \mathrm{O}
$$




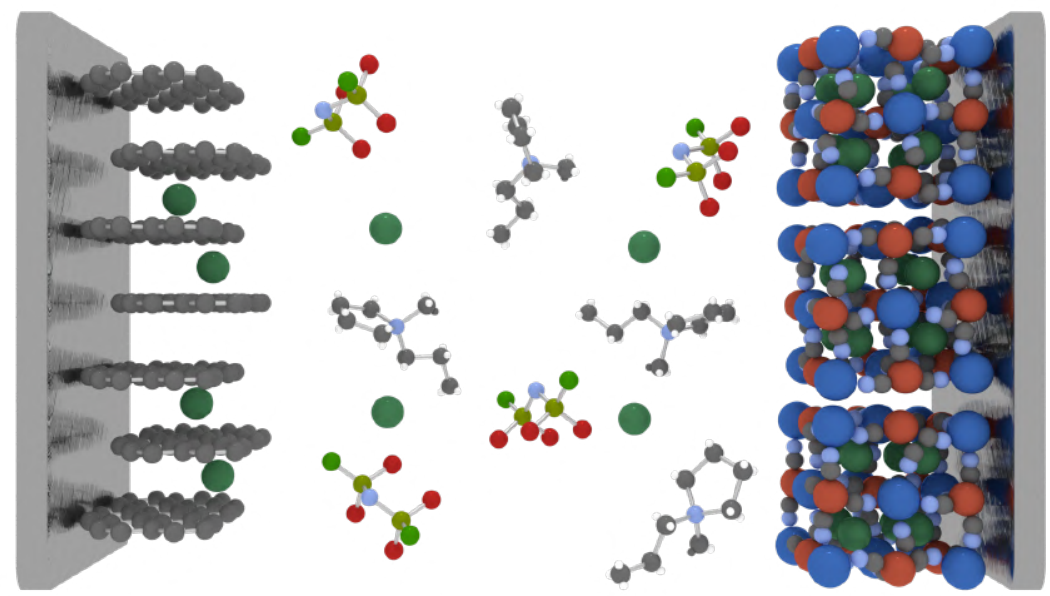

Figure 1: A schematic of the full-cell potassium-ion chemistry consisting of a graphite anode, potassium bis(fluorosulfonyl)imide in N-butyl-N-methylpyrrolidinium bis(fluorosulfonyl)imide electrolyte, and potassium manganese hexacyanoferrate cathode.

in which $\mathrm{A}$ is the inserting alkali metal, $\mathrm{M}$ and $\mathrm{M}^{\prime}$ are the two transition metals in oxidation states $\mathrm{J}$ and $\mathrm{K}$ respectively, $\mathrm{y}$ is the fraction of hexacyanometallate vacancies, and $\mathrm{w}$ is the number of water molecules per formula unit. Research into cathode materials have focused on the hexacyanoferrates because of their high potential on ion insertion $^{13}$ and a diverse choices of nitrogen-coordinated elements have been studied. ${ }^{14-17}$ Potassium manganese hexacyanoferrate (KMF) is the most attractive composition for high energy density KIBs due to the high potential of the $\mathrm{Mn}$ (III/II) redox couple at around $4.1 \mathrm{~V}$ vs $\mathrm{K}^{+} / \mathrm{K}$ and its high theoretical specific capacity of $155 \mathrm{~mA} \mathrm{~h} \mathrm{~g}^{-1} \cdot{ }^{17-20}$

For a viable KMF | graphite battery it is essential that the electrolyte is compatible with the graphite anode, which broadly prevents the use of $\mathrm{KPF}_{6}$ salt $^{8,21}$ or fluoroethylene carbonate (FEC) additive. ${ }^{19}$ Current KMF literature, that fulfil this requirement, suffer from impractically low coulombic efficiencies $(\approx 95 \%)$ caused by electrolyte instability at high potential and corrosion of the aluminium current collector. ${ }^{19,20,22}$ Increasing the coulombic efficiency is crucial for practical battery chemistries. ${ }^{23}$

In this study, we make two key advancements to improve the performance of KMF, with a focus on addressing low coulombic efficiency. First, we employ a high-throughput multi-pipetting robot to explore the effect of synthesis conditions on KMF particle size and defect content. We achieve fine control of particle size whilst reducing vacancy and water content to lowest reported levels. Second, we use an ionic liquid electrolyte. Rather than attempting to increase the stability of an inherently reactive solvent, we use an intrinsically stable electrolytic solution: $1 \mathrm{~mol} \mathrm{~kg}^{-1}$ potassium bis(fluorosulfonyl)imide (KFSI) in the ionic liquid N-butyl-N-methylpyrrolidinium bis(fluorosulfonyl)imide ( $\left.\mathrm{Pyr}_{1,3} \mathrm{FSI}\right)$. During electrochemical testing we observe clear relationships between particle size, specific capacity, and coulombic efficiency for KMF materials. The optimised KMF material achieved a stable coulombic efficiency of $99.3 \%$, a significant improvement over the previous best of $\approx 95 \%$. In the same electrolyte graphite displays excellent cyclability and rate performance, equivalent to or better than the current state of the art.

\section{High-throughput optimization and detailed characterization}

The KMF cathode material was optimized by highthroughput synthesis and characterization. Three synthesis parameters that have been shown to influence PBA morphology in previous studies were explored systematically for the first time in this work. Those parameters are the concentration of the presusors manganese(II) chloride and potassium hexacyanoferrate $\left(\left[\mathrm{MnCl}_{2}\right]=[\mathrm{Mn}]=\left[\mathrm{K}_{4}\left[\mathrm{Fe}(\mathrm{CN})_{6}\right] \in\right.\right.$ $\{25,75,125\} \mathrm{mM})$, the ratio of potassium citrate to manganese chloride ${ }^{24,25}$ ([citrate]: $[\mathrm{Mn}] \in\{1,2.5$, $5,10\})$, and the presence of potassium chloride ${ }^{26}$ $([\mathrm{KCl}] \in\{0,1.5\} \mathrm{M})$. The upper limits of the ranges were chosen as a compromise between the solubility limits of potassium citrate, potassium hexacyanoferrate, and potassium chloride. The search space of synthesis conditions is mapped in Figure 2a.

The effect of these parameters on three physical properties, crystal structure, particle size, and vacancy content, was measured. The crystal structures of all 24 materials were measured by XRD. Diffractograms of the materials on the 8 corners of 
a)

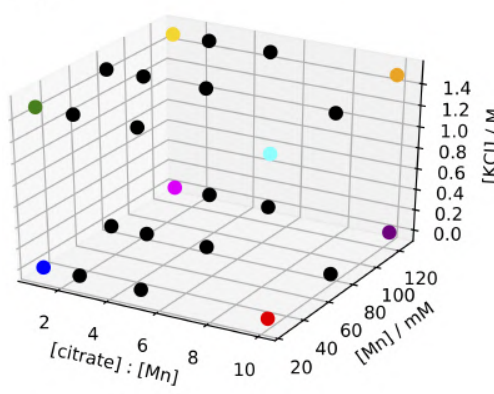

d)

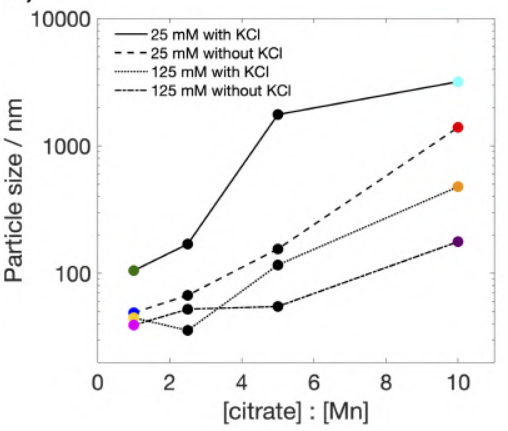

b)

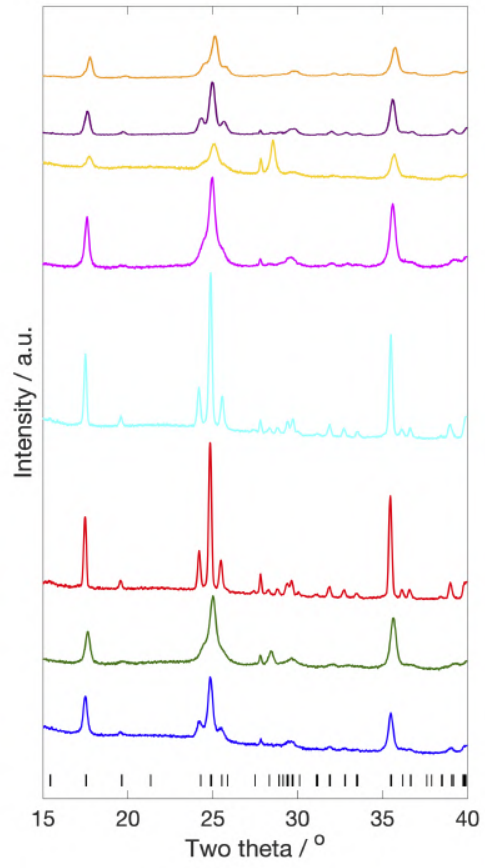

C)

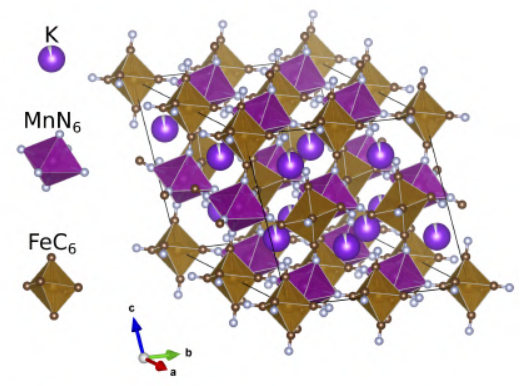

e)

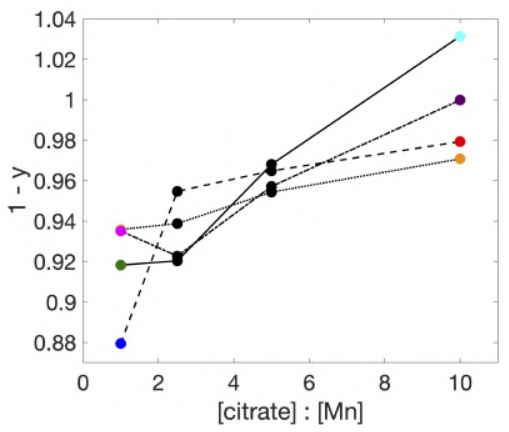

Figure 2: High-throughput synthesis and characterization of KMF. (a) Search space for the synthesis parameters. (b) Diffractograms of the materials at the 8 corners of the search space. (c) Schematic of the crystal structure. (d) Effect of the ratio of citrate to manganese on the materials particle size, as determined by analysis of the electron micrographs (see Supplementary Figures 1-3 and Supplementary Table 1 for the full data set). (e) Effect of the ratio of citrate to manganese on vacancy content, as determined by ICP-OES (see Supplementary Table 1 for the full data set).

the search space are plotted in Figure 2b. The crystal structures of all 24 materials have monoclinic geometries and are members of the $P 2_{1} / c$ space group, as shown in Figure 2c. Results of Rietveld refinement are presented in Supplementary Table 1. The unit-cell volumes range from 514.3 to $519.5 \AA^{3}$. These results are consistent with previous XRD studies of KMF materials. ${ }^{19}$ Peak broadening was more significant for samples at higher reactant concentrations, lower citrate concentrations, and in the presence of potassium chloride. We attribute this peak broadening to decreasing particle size.

This conclusion is supported by particle size analysis of SEM images, Figure 2d (SEM images in Supplementary Figures 1-3). Most samples had particle sizes below $100 \mathrm{~nm}$, and only four samples had particle sizes above $1000 \mathrm{~nm}$. Finally, the vacancy content of the samples were determined by ICP-OES. The full ICP-OES results are presented in Supplementary Table 1, and the vacancy contents are plotted in Figure 2e. The samples with the highest fraction of $\left[\mathrm{Fe}(\mathrm{CN})_{6}\right]$ octahedra (and therefore the lowest vacancy content) were those synthesized in the highest citrate concentration. At a citrate-to-manganese ratio of 10 , all samples had a vacancy content below $5 \%$. Based on these highthroughput results, four materials with monoclinic geometry, minimal vacancy content, and a range of particle sizes were selected for further study. A summary of results from high-throughput analysis can be found in Supplementary Table 1.

These four materials were synthesized in larger batches under the conditions listed in Table 1. SEM micrographs of these 4 materials are shown in Figure $3 \mathrm{a}-\mathrm{d}$, with particle sizes, measured from the micrographs, of approximately 39, 55, 177, and $297 \mathrm{~nm}$, respectively. The materials are designated $K M F_{39}, K M F_{55}, K M F_{177}$, and $K M F_{297}$. They are approximately cubic in shape and the smallest particles experience significant agglomeration.

High-resolution synchrotron XRD patterns were taken and the data refined according to the Rietveld method. The diffractograms, shown in Figure 3e, confirm the monoclinic geometry and $P 2_{1} / c$ space group observed in the high-throughput step and also reported elsewhere. ${ }^{17}$ (Full diffraction patterns, Rietveld refinement residuals, and refined parameters are available in Supplementary Figures 4-7 and Supplementary Tables 2-5.) Distortion and densification of this phase are driven by displacement of the potassium atoms from the center of the subcube, causing rotation of the $\mathrm{FeC}_{6}$ and $\mathrm{MnN}_{6}$ octahedra and deviation of the bond angles away from $180^{\circ}$, Figure 2c. The lattice parameters are presented in Table 1 with the compositions, as determined by ICP-OES. 
a)

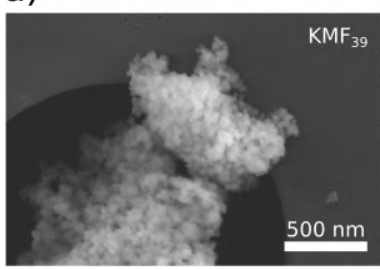

e)

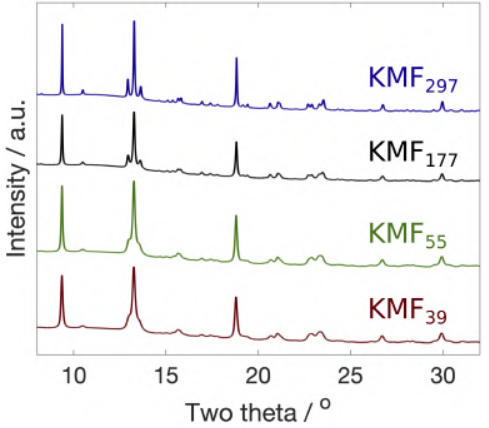

b)

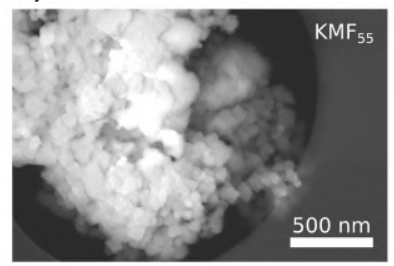

f)

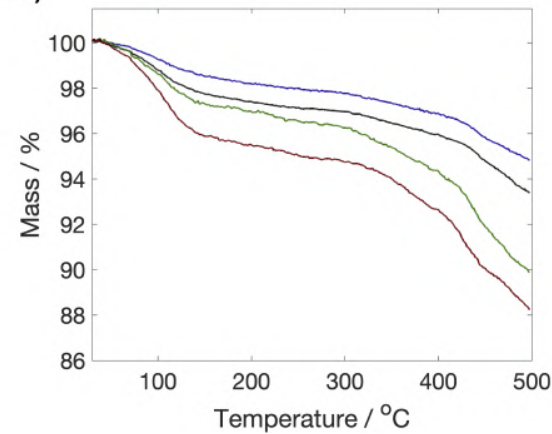

d)
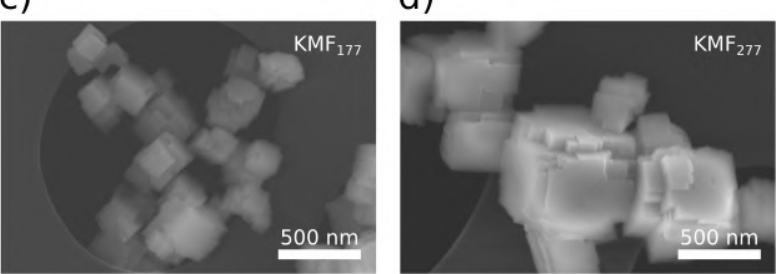

g)

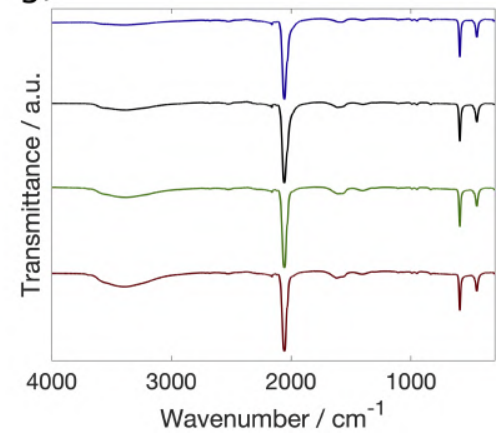

Figure 3: Characterisation of the four selected KMF materials. (a-d) SEM micrographs of $K M F_{39}, K M F_{55}$, $K M F_{177}$, and $K M F_{297}$ respectively. (e) Synchrotron XRD, (f) FTIR, and (g) TGA of the four selected materials. Results from the Rietveld refinement of patterns in (e) can be found in Supplementary Table 1.

\begin{tabular}{cccccccccccc}
\hline Sample & $\begin{array}{c}{[\mathrm{Mn}] /} \\
\mathrm{mM}\end{array}$ & $\begin{array}{c}{[\mathrm{citrate}]:} \\
{[\mathrm{Mn}]}\end{array}$ & $\begin{array}{c}{[\mathrm{KCl} /} \\
\mathrm{M}\end{array}$ & $\mathrm{d} / \mathrm{nm}$ & $\mathrm{x}$ & $1-\mathrm{y}$ & $\mathrm{w}$ & $\mathrm{a} / \AA$ & $\mathrm{b} / \AA$ & $\mathrm{c} / \AA$ & $\beta /{ }^{\circ}$ \\
\hline $\mathrm{KMF}_{39}$ & 125 & 1 & 1.5 & 39 & 1.92 & 0.95 & 0.78 & $6.98842(20)$ & $7.29052(18)$ & $12.27740(4)$ & $124.6312(17)$ \\
\hline $\mathrm{KMF}_{55}$ & 125 & 5 & 1.5 & 55 & 1.86 & 0.94 & 0.52 & $6.98188(17)$ & $7.28117(16)$ & $12.25202(32)$ & $124.5931(15)$ \\
\hline $\mathrm{KMF}_{177}$ & 125 & 10 & 1.5 & 177 & 1.91 & 0.97 & 0.43 & $6.97207(8)$ & $7.32166(7)$ & $12.26838(13)$ & $124.4862(6)$ \\
\hline $\mathrm{KMF}_{297}$ & 75 & 10 & 1.5 & 297 & 1.89 & 0.98 & 0.28 & $6.96126(8)$ & $7.33250(7)$ & $12.26209(15)$ & $124.4726(6)$ \\
\hline
\end{tabular}

Table 1: Synthesis parameters of the four selected materials and results of their characterisation including particle size 'd', composition values ' $\mathrm{x}$ ' ' $1-\mathrm{y}$ ' ' $w$ ', and lattice parameters ' $a$ ' 'b' 'c' and ' $\beta$ '.

All the materials were highly potassiated as synthesized, with at least 1.85 potassium atoms per formula unit. This indicates both low vacancy, confirmed by the ratio $[\mathrm{Fe}]:[\mathrm{Mn}]=1-\mathrm{y}$ which is at least $94 \%$ for all samples, as well as a low oxidation state, which is predominantly +2 for both iron and manganese. TGA of the 4 samples show mass losses of between about $2 \%$ and $5 \%$ from room temperature up to a decomposition temperature of between about 350 and $400^{\circ} \mathrm{C}$ (Figure $3 \mathrm{f}$ ). This corresponds to a loss of surface-absorbed water, zeolitic water (present in the interstices), and water coordinated to manganese at vacancies of the hexacyanoferrate octahedra. The water contents per formula unit are presented alongside the ICP-OES data in Table 1. Water content increases from 0.28 to 0.78 with decreasing particle size. The relationship is due in part to the fact that smaller particles have larger surface-area-to-volume ratios, and because smaller particles have higher vacancy contents. This convolution of causes is well known in the literature ${ }^{27}$ and more careful study to understand the separate effects is warranted.

FTIR data agree with the size-to-water-content trend. The spectra, shown in Figure 3g, contain two peaks arising from water: a broad peak between 3100 and $3600 \mathrm{~cm}^{-1}$ and a sharper peak at about $1650 \mathrm{~cm}^{-1}$. Both of these decrease in intensity as the samples increase in particle size. Sharp peaks at 2057,592 , and $449 \mathrm{~cm}^{-1}$ correspond to the framework vibrations $v(\mathrm{CN}), \delta(\mathrm{FeCN})$, and $v(\mathrm{FeC})$ respectively. The structural distortion of the materials away from cubic, observed in the synchrotron XRD data, effects slightly different environments for the Fe-CN-Mn bonds. This gives rise to the shoulder observed on the peak at $2057 \mathrm{~cm}^{-1}$. 
a)

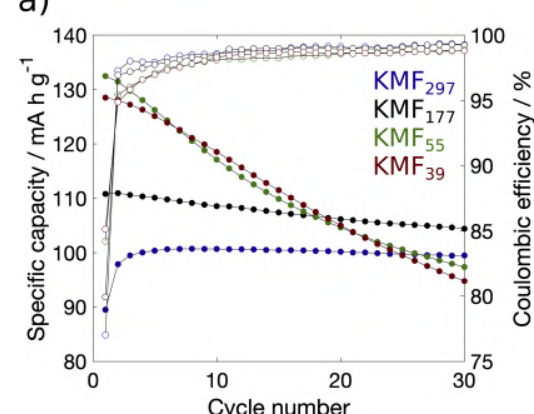

d)

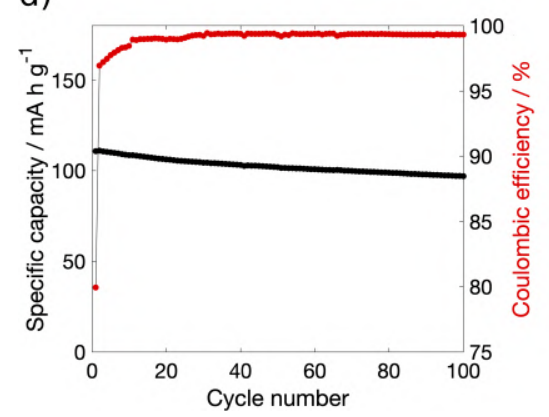

b)

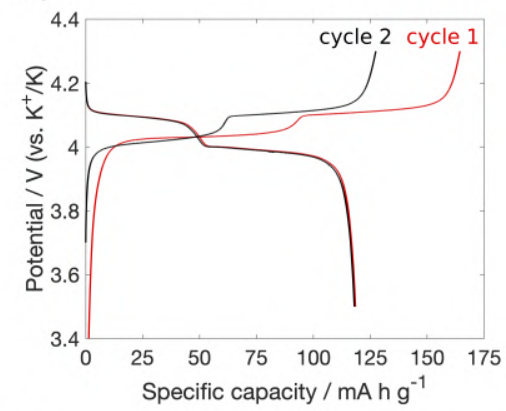

e)

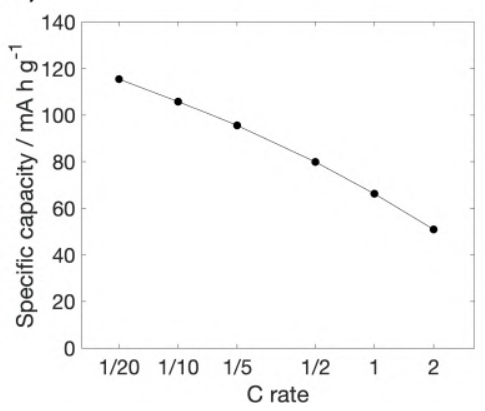

c)

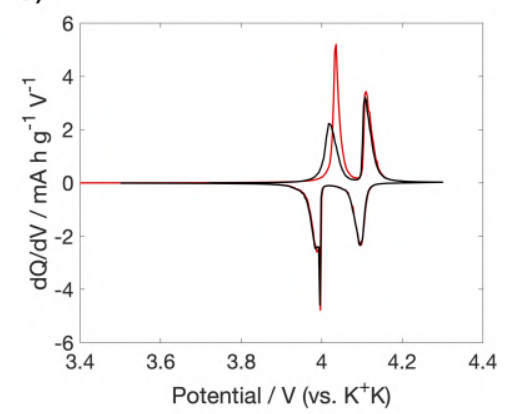

f)

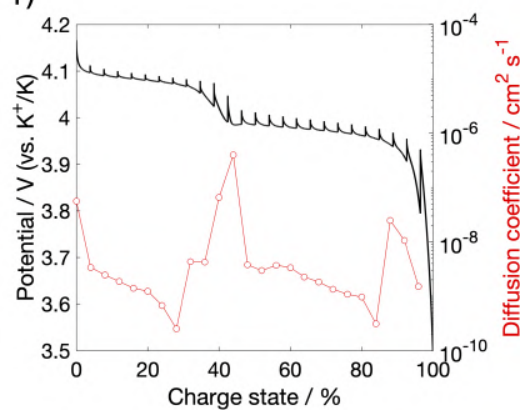

Figure 4: Galvanostatic cycling results for the KMF cathode. (a) Discharge capacity and coulombic efficiency at $\mathrm{C} / 10$ for the selected KMF materials, from the smallest particle size to the biggest: $K M F_{39}, K M F_{55}, K M F_{177}$, and $K M F_{297}$. (b) and (c) the first and second cycle galvanostatic profile and differential capacity of $K M F_{177}$. (d) Capacity retention of $K M F_{177}$ over the first 100 cycles at C/10 in a two-electrode configuration. (e) rate capability of $K M F_{177}$ at $\mathrm{C}$ rates from C/20 to 2C. (f) Galvanostatic intermittent titration technique (GITT) of $K M F_{177}$ on a discharge.

\section{Electrochemistry of the optimized manganese hexacyanoferrates}

The ionic liquid electrolyte, $1 \mathrm{~mol} \mathrm{~kg}^{-1}$ KFSI in $\mathrm{Pyr}_{1,3}$ FSI, was used for all electrochemical measurements. At $30^{\circ} \mathrm{C}$ the electrolyte had an ionic conductivity of $3.312 \mathrm{mS} \mathrm{cm}^{-1}$ with an activation energy of $9.30 \mathrm{~kJ} \mathrm{~mol}^{-1}$, and viscosity of $71.7 \mathrm{mPas}$. Temperature dependent conductivity and viscosity plots can be found in Supplementary Figure 8. Linear sweep voltammetry (LSV), Supplementary Figure 9, with an aluminium foil working electrode was performed to study the corrosion resistance and electrolyte stability at high potentials. Scanning up to $5 \mathrm{~V}$ produced a current density of less than $10 \mu \mathrm{A} \mathrm{cm}^{-2}$, which decreases substantially on subsequent cycles. The electrolyte was shown to be thermally stable up to approximately $250^{\circ} \mathrm{C}$, Supplementary Figure 10.

Particle size has been shown to affect the electrochemical performance of battery electrode materials generally and of PBA cathodes in particular. ${ }^{15,27}$ After high-throughput selection of 4 promising KMF candidates and thorough materials characterization, the 4 samples were subjected to electrochemical tests in a half-cell configuration to select the best performing to test in a full-cell configuration. The samples were cycled at a rate of
$\mathrm{C} / 10$ in a coin cell with a potassium counter electrode and the ionic liquid electrolyte, $1 \mathrm{~mol} \mathrm{~kg}^{-1}$ KFSI in $\operatorname{Pyr}_{1,3}$ FSI. The specific capacity versus cycle number for the samples is plotted in Figure 4a. Ordered by first-cycle specific capacities, the materials are $K M F_{55}\left(133 \mathrm{~mA} \mathrm{~h} \mathrm{~g}^{-1}\right) K M F_{39}$ $\left(129 \mathrm{mAhg}^{-1}\right)>K M F_{177}\left(111 \mathrm{mAhg}^{-1}\right)>$ $K M F_{297}\left(90 \mathrm{~mA} \mathrm{~h} \mathrm{~g}^{-1}\right.$, though it quickly increases to $100 \mathrm{~mA} \mathrm{~h} \mathrm{~g}^{-1}$, possibly due to a surface activation process $\left.{ }^{19,28}\right)$. These data suggest that the decrease in specific capacity is a kinetic effect, and that at some particle size above $55 \mathrm{~nm}$ either the electronic or ionic conductivity of the material limit the potassium-insertion reaction.

The cyclability of the samples, though, is much greater for the larger particles. In fact, the trend in capacity retention after 30 cycles (30th cycle discharge capacity compared to the maximum) is exactly the opposite to that of first-cycle specific capacity: $K M F_{297}(98.8 \%)>K M F_{177}(94.1 \%)$ $>K M F_{39}(73.7 \%) \approx K M F_{55}(73.5 \%)$. While capacity fade in PBA electrodes has been attributed to numerous factors including phase transformations, manganese(III) disproportionation, cathode dissolution, and parasitic reactions with water, the correlation with particle size suggests in this case the decreasing capacity stems from surface-specific phenomena. The improved cyclability of $K M F_{297}$ and $K M F_{177}$ are reflected in their coulombic effi- 
a)

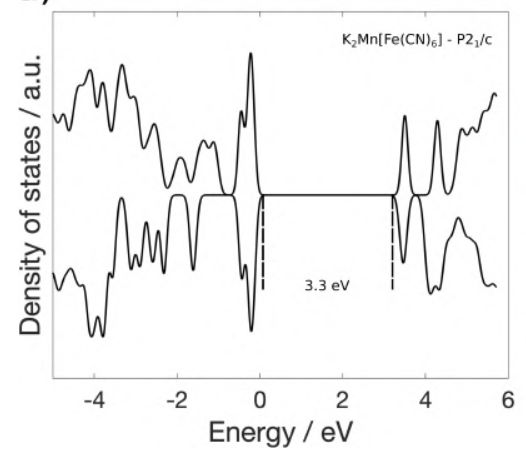

b)

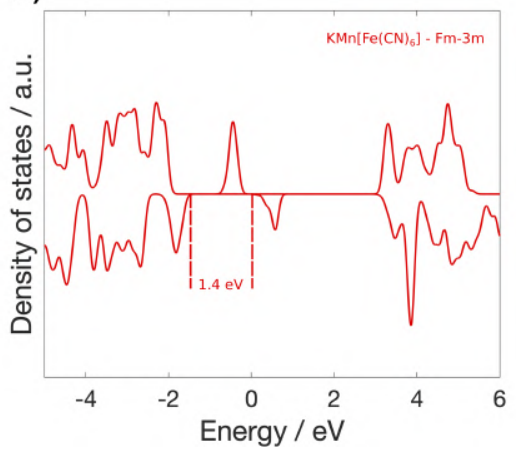

c)

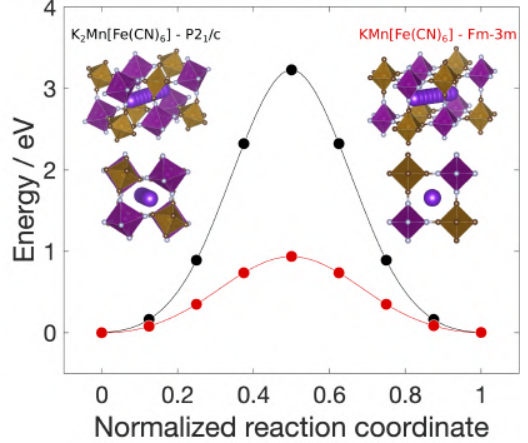

Figure 5: Density functional theory (DFT) calculations. (a) and (b) density of states (DOS) plots of $\mathrm{K}_{2} \mathrm{Mn}\left[\mathrm{Fe}(\mathrm{CN})_{6}\right]$ and $\mathrm{KMn}\left[\mathrm{Fe}(\mathrm{CN})_{6}\right]$. (c) Activation energy for potassium migration in both $\mathrm{K}_{2} \mathrm{Mn}\left[\mathrm{Fe}(\mathrm{CN})_{6}\right]$ and $\mathrm{KMn}\left[\mathrm{Fe}(\mathrm{CN})_{6}\right]$ structures. Insets show the migration path modelled in each case.

ciencies, plotted in Figure 4a along with those of the other two samples. $K M F_{177}$ in particular rises to a coulombic efficiency of $99.3 \%$ by the 25 th cycle. As far as the authors can determine, this is the highest coulombic efficiency for a KMF cathode material, except for those that include FEC in the electrolyte which is incompatible with a graphite anode. ${ }^{28}$

$K M F_{177}$ was selected as a compromise between specific capacity and coulombic efficiency for more thorough investigation in a three-electrode cell with potassium metal as the counter and reference electrodes. The electrolyte was kept the same. The galvanostatic profiles and differential capacities of the first two cycles are plotted in Figure 4b-c. They show two plateaus. The lower plateau, corresponding to the $\mathrm{Fe}(\mathrm{III} / \mathrm{II})$ redox couple, is found at $3.99 \mathrm{~V}$ and $4.04 \mathrm{~V}$ on potassiation and depotassiation, respectively. The upper plateau, corresponding to the $\mathrm{Mn}(\mathrm{III} / \mathrm{II})$ redox couple, is found at $4.10 \mathrm{~V}$ and $4.11 \mathrm{~V}$ on potassiation and depotassiation, respectively. The average potentials are therefore $V_{\text {cat }}$ $=4.02 \mathrm{~V}$ and $V_{\text {and }}=4.08 \mathrm{~V} \cdot{ }^{29}$ Over the first 100 cycles $K M F_{177}$ retains $87.4 \%$ of the first-cycle specific capacity and the coulombic efficiency stabilizes above $99.3 \%$, Figure 4d. Figure 4e, plotting the rate performance, shows the material has a specific capacity of $119 \mathrm{~mA} \mathrm{~h} \mathrm{~g}^{-1}$ at a $\mathrm{C}$ rate of $\mathrm{C} / 20$. It retains $89 \%$ of this specific capacity at $\mathrm{C} / 10,80 \%$ at $\mathrm{C} / 5,67 \%$ at $\mathrm{C} / 2,56 \%$ at $1 \mathrm{C}$, and $43 \%$ at $2 \mathrm{C}$. See Supplementary Figure 11 for the corresponding differential capacity plots. All potentials are given versus the $\mathrm{K}^{+} / \mathrm{K}$ redox couple. The half-cell performance of $K M F_{177}$ is compared to other candidate K-ion cathodes in Supplementary Table 6.

The sluggish kinetics shown by KMF is consistent with other reports ${ }^{15,27}$ but contrary to sodium manganese hexacyanoferrate ${ }^{30}$ and KMF operated in aqueous electryoltes. ${ }^{18}$ One possible explanation is that the large potassium ion has slow bulk diffusion through the distorted channels when not mediated by water in the structure. The diffusion coefficient calculated from galvanostatic intermittent titration technique (GITT) on $K M F_{177}$, Figure $4 \mathrm{f}$, is in the order of $10^{-9} \mathrm{~cm}^{2} \mathrm{~s}^{-1}$ over the whole state of charge.

The kinetics of the insertion reaction were investigated by density functional theory (DFT). The band gaps $\left(E_{g}\right)$ and the diffusion energy barrier $\left(E_{a}\right)$ of two oxidation states for the material were calculated and compared. Figure 5a and b show that the band gap increases from $1.4 \mathrm{eV}$ to $3.3 \mathrm{eV}$ on reduction from cubic $\mathrm{KMn}\left[\mathrm{Fe}(\mathrm{CN})_{6}\right]$ to monoclinic $\mathrm{K}_{2} \mathrm{Mn}\left[\mathrm{Fe}(\mathrm{CN})_{6}\right]$, suggesting a lower electronic conductivity for the fully reduced material. At the same time, the activation energy for diffusion of potassium ions increases from $0.93 \mathrm{eV}$ to $3.2 \mathrm{eV}$ for the reduced and intermediate oxidation states, respectively. This implies a decrease in the ionic conductivity. While the change to the diffusion barrier is larger, these data alone cannot determine whether the slow down to ionic or electronic conductivity is primarily responsible for the sluggish kinetics observed experimentally. However, these calculations together do show that the kinetics of the insertion reaction slow significantly after the material is sufficiently potassiated to induce the phase change from the cubic to the monoclinic geometry. Further calculations are needed to determine whether the changes to the band gap and diffusion barrier are a primarily electronic (that is, caused by the oxidation state) or structural (that is, caused by the crystal geometry and lattice parameter) effect.

\section{Electrochemistry of a graphite anode and a potassium-ion full cell}

To understand the feasibility of pairing this optimized KMF cathode with a graphite anode for a useful full-cell battery, we studied the electro- 
a)

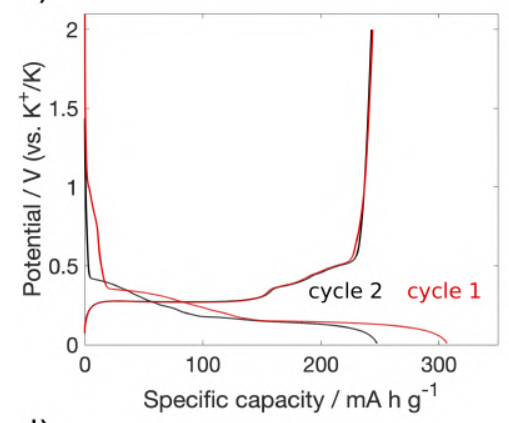

d)

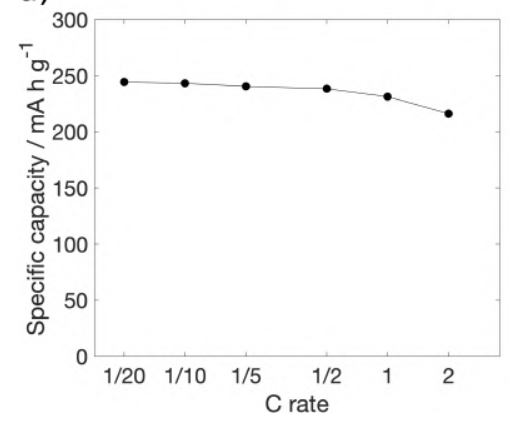

b)

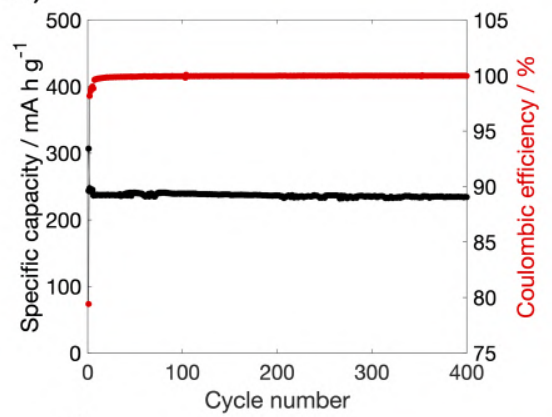

e)

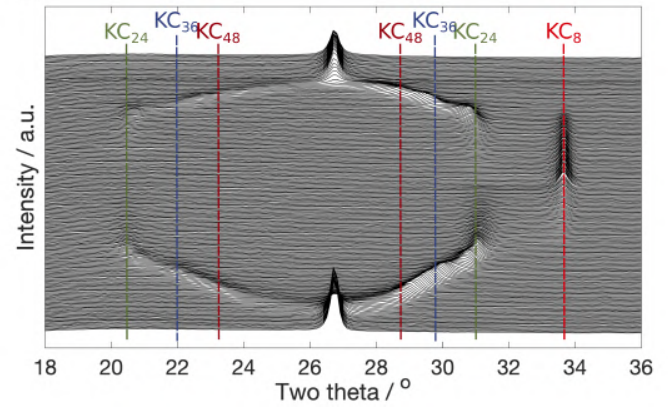

c)

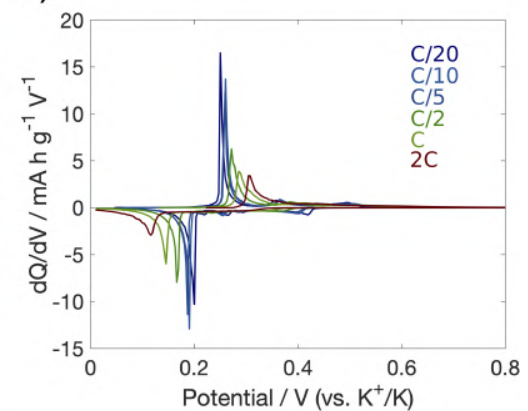

f)

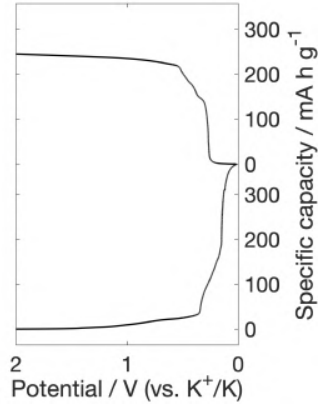

Figure 6: Galvanostatic cycling results for the graphite anode. (a) First and second cycle galvanostatic profiles of graphite. (b) Capacity retention and coulombic efficiency for 400 cycles (cycles $1-5$ at C/20, 6-400 at C/5). (c) and (d) differential capacity and capacity retention of graphite at $\mathrm{C}$ rates from $\mathrm{C} / 20$ to $2 \mathrm{C}$. (e) and (f) Operando $\mathrm{X}$-ray diffraction patterns, and relative potential versus capacity profiles for the potassium intercalation and de-intercalation into graphite at $\mathrm{C} / 30$. The major peaks for the intermediates $\mathrm{KC}_{48}, \mathrm{KC}_{36}, \mathrm{KC}_{24}$ and $\mathrm{KC}_{8}$ are highlighted.

chemical intercalation of $\mathrm{K}^{+}$into graphite in the same ionic-liquid electrolyte. Figure 6 a shows the galvanostatic charge and discharge profiles of the first two cycles. The galvanostatic profiles have several small steps, with the longest plateau located between 0.20 and $0.25 \mathrm{~V}$ versus the $\mathrm{K}^{+} / \mathrm{K}$ redox couple on depotassiation and potassiation, respectively. Figure $6 \mathrm{~b}$ shows the high cyclability of the graphite anode. After 5 cycles of formation of a solid-electrolyte interphase (SEI), the graphite achieves a specific capacity of $235 \mathrm{~mA} \mathrm{~h}^{-1}$ at a rate of $\mathrm{C} / 5$. It maintains over $99 \%$ of this specific capacity after 400 cycles with an average coulombic efficiency of over $99.94 \%$.

The increase in overpotential as the rate is increased is shown in differential capacity plots in Figure 6c. Increasing the rate from $\mathrm{C} / 20$ to $2 \mathrm{C}$ led to an increase of less than $100 \mathrm{mV}$ in overpotential. The graphite has a specific capacity of $246 \mathrm{~mA} \mathrm{~h}^{-1}$ at $\mathrm{C} / 20$, and retains $99 \%$ of this specific capacity at $\mathrm{C} / 10,98 \%$ at $\mathrm{C} / 5,97 \%$ at $2 \mathrm{C}, 94 \%$ at $1 \mathrm{C}$, and $88 \%$ at $2 \mathrm{C}$. These data are plotted in Figure 6d. We also probed how the graphite crystal structure changes during the intercalation reaction by operando XRD. We found that the multiple steps observed in the galvanostatic charge-discharge profile can be explained by phase changes in the graphite during intercalation, in agreement with previous studies. ${ }^{21,31}$ The diffractograms taken during the course of the electrochemical reaction are overlaid and plotted next to the accompanying specific capacity versus potential plot in Figures 6e-f. We match various potassium-graphite stoichiometries with stage-n graphite intercalation compounds, where the index $\mathrm{n}$ is the number of graphene layers between intercalation planes. ${ }^{31}$ In the first potassiation region, between $2.00 \mathrm{~V}$ and $0.33 \mathrm{~V}$, changes in the crystal structure are negligible, indicating that electrolyte decomposition (or a similar reaction), rather than graphite intercalation, is occurring. Between $0.33 \mathrm{~V}$ and $0.20 \mathrm{~V}$ two new phases are found: first $\mathrm{KC}_{48}$ (stage-4) and second $\mathrm{K}_{36}$ (stage-3). Between $0.20 \mathrm{~V}$ and $0.15 \mathrm{~V}$ we observe $\mathrm{KC}_{24}$ (stage-2). Finally, below $0.15 \mathrm{~V}$, we find the fully potassiated phase $\mathrm{KC}_{8}$ (stage-1) indicated by its strong peak at an angle of $33.5^{\circ}$. We then electrochemically removed the potassium and observed the anode revert through the same series of phases back to graphite. We recovered the (002) peak of pure graphite without the shoulder observed in previous reports. ${ }^{21,32}$

The performance of a KMF | KFSI in $\mathrm{Pyr}_{1,3} \mathrm{FSI}$ | graphite full cell was tested in three-electrode configuration with a potassium reference. Figure 7 a shows the full cell voltage profile during the first five cycles. For stable cycling it was important that the full cell was limited by the graphite anode as it ensured an effective SEI was formed. Full 
a)

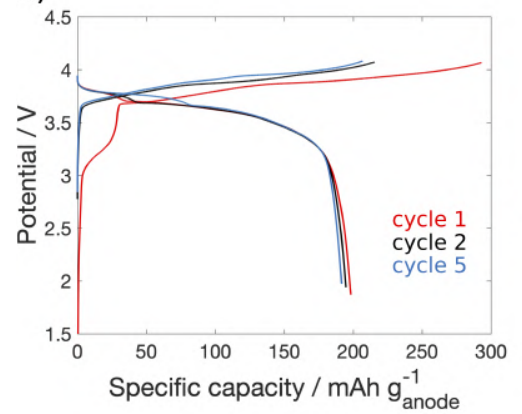

b)

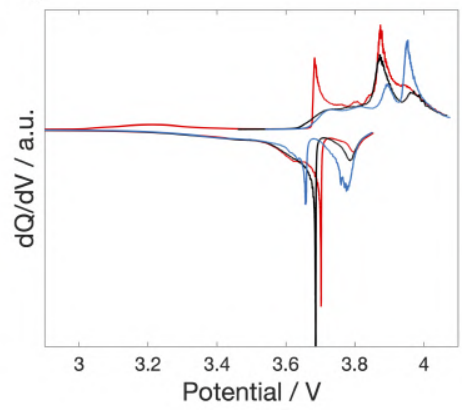

C)

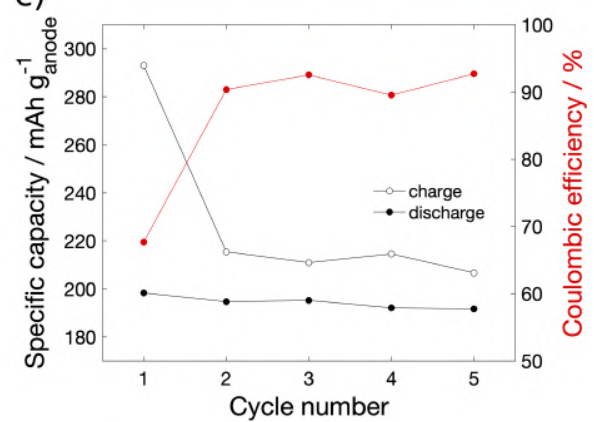

Figure 7: KMF | KFSI in $\mathrm{Pyr}_{1,3}$ FSI | graphite full cell cycling results. (a) and (b) the first, second and fifth galvanostatic profiles and differential capacity plots for the full cell cycled at C/10. (c) Specific capacity and coulombic efficiency of the full cell. The mass ratio of KMF:graphite was 3.6:1.

cells with excess anode suffered significant capacity loss. Supplementary Figure 12 shows the cathode and anode potentials with respect to the potassium reference electrode. The average discharge voltage was around $3.6 \mathrm{~V}$ and dependent on the start and finish charge state of the KMF cathode. Differential capacity plot, Figure $7 \mathrm{~b}$, shows how the low coulombic efficiency of the cell causes a shift in the state of charge over which the KMF cathode operates. This is detrimental to the cyclability of the full cell and further optimisation, possibly by addition of electrolyte additives, is required to enable stable long term cycling at slow rates. The full cell had a first cycle coulombic efficiency of $67.7 \%$ and a discharge specific capacity of $198 \mathrm{~mA} \mathrm{~h} \mathrm{~g}^{-1}$ (normalised to the anode), Figure 7c.

\section{Conclusions}

In summary, through optimisation of the KMF morphology and by using an ionic liquid electrolyte the coulombic efficiency of both KMF cathode and conventional graphite anode was significantly improved, providing a route to highly efficient highvoltage potassium-ion batteries.

Using an automated pipetting robot and highthroughput characterization techniques a wide KMF synthesis parameter space was studied. We found that the addition of citrate can increase particle sizes from $<100 \mathrm{~nm}$ to $>1000 \mathrm{~nm}$ and decreases vacancy content. Simultaneous addition of $\mathrm{KCl}$ also decreases vacancy content but also decreases particle size. Decreasing the reactant concentration increases the crystallinity of the material. We also observed a clear negative correlation between particle size and water content. Electrochemical testing in a KFSI in $\mathrm{Pyr}_{1,3} \mathrm{FSI}$ ionic liquid electrolyte showed that an intermediate particle size of about $180 \mathrm{~nm}$ is an optimal compromise between high specific capacity $\left(119 \mathrm{~mA} \mathrm{~h}^{-1}\right)$ and long cycle life $(87.4 \%$ retention after 100 cycles) at about $4 \mathrm{~V}$ versus $\mathrm{K}^{+} / \mathrm{K}$. We also report a coulombic effi- ciency (an important but severely underappreciated figure of merit) of above $99.3 \%$ - the highest for a PBA potassium-ion cathode of which we are aware. This improvement is attributed to the stability of the ionic liquid electrolyte at high voltages and suppression of aluminium current collector corrosion.

In the same electrolyte graphite displayed excellent cyclability (retaining $99 \%$ of its initial capacity after 400 cycles) and rate performance (maintaining $88 \%$ of its $\mathrm{C} / 20$ capacity at $2 \mathrm{C}$ ). We observed structural changes in graphite by operando XRD, and we recovered, for the first time, phase-pure depotassiated graphite. Finally, we achieved the highest first-cycle coulombic efficiency of a KMF | KFSI in $\mathrm{Pyr}_{1,3}$ FSI | graphite full cell of $67.7 \%$. This work shows that the use of an ionic-liquid electrolyte in a KMF | graphite potassium-ion full cell is a viable method of minimizing irreversible capacity loss, which is critical to fabricating useful, energy-dense potassium-ion batteries. Future work focussing on overcoming causes of poor kinetics in $\mathrm{KMF}$ and strategies to minimise surface reactions is merited and will further improve full cell performance.

\section{Methods}

High throughput synthesis: Automated synthesis was performed using a Hamilton Microlab NIMBUS multi-pipetting robot with four pipetting channels. Materials were synthesised in glass vials in 24-well plate made from aluminium on a heater-stirrer plate. For each sample, the robot prepared a mixture of manganese(II) chloride (Acros) and potassium citrate (Sigma-Aldrich); prepared, in a separate vial, a mixture of potassium hexacyanoferrate (Sigma-Aldrich) and potassium chloride (Scientific Laboratory Supplies), as appropriate; heated the mixtures to $70^{\circ} \mathrm{C}$ and began mixing by shaking; added the mixture of manganese(II) chloride and potassium citrate dropwise to the hexacyanoferrate. Samples were capped and allowed to mature for $1 \mathrm{~h}$ 
at $70^{\circ} \mathrm{C}$. All samples were washed with deionized water; phase-separated in a centrifuge; and dried in atmosphere overnight at $80^{\circ} \mathrm{C}$.

Materials characterization: Elemental composition and thermal analysis coupled with mass spectrometry were performed by inductively coupled plasma optical emission spectrometry (Perkin Elmer Optima 8000) and thermogravimetric analysis mass spectrometry (NETZSCH STA 449 F3 Jupiter coupled to a QMS 403 Aeolos). Scanning electron microscopy was carried out on a FE-SEM (Zeiss Merlin). Particle size analysis of SEM images was performed using ImageJ. Synchrotron X-ray diffraction was performed at beamline I11 at Diamond Light Source on the position sensitive detector (PSD). Laboratory X-ray diffraction was performed on a benchtop XRD (RIGAKU miniflex). Synchrotron XRD was refined using GSAS and high-throughput laboratory data was refined in a simultaneous manner using GSAS 2. Fourier transform infrared spectroscopy (Thermo Scientific Nicolet 6700) was performed in attenuated reflectance (ATR) mode in a nitrogen filled glove box.

Electrochemical Tests: The graphite (SFG6, TIMCAL) was firstly ball milled for $3 \mathrm{~h}$ at 200 rpm (Restch PM100) to improve its rate capability and capacity retention. ${ }^{33}$ The carbon based working electrode was then prepared mixing the graphite with poly(acrylic acid) $\left(\mathrm{M}_{w} \approx 450,000 \mathrm{Da}\right.$, Sigma-Aldrich) in the weight ratio of 9:1 respectively. This mixture was dispersed in 1-methyl-2pyrrolidone (NMP) to prepare the slurry and cast on aluminum foil using a doctor blade. The casting was dried under vacuum at $80^{\circ} \mathrm{C}$ overnight and then roll-pressed. The KMF working electrodes were prepared in a similar way, but the active material was mixed with SuperP carbon (TIMCAL Carbon Black Super P) and poly(vinylidene fluoride) (MTI corp, $\mathrm{M}_{w} 600,000 \mathrm{Da}$ ) in the weight ratio of $7: 2: 1$. The slurry was cast on carbon-coated aluminum. Disks of $10 \mathrm{~mm}$ diameter were punched out, and the active material loading was 1.5 to $2.0 \mathrm{mg} \mathrm{cm}^{-2}$.

The electrolyte used was a $1 \mathrm{~mol} \mathrm{~kg}^{-1}$ solution of potassium bis(fluorosulfonyl)imide (KFSI, 99.9\% Solvionic) in N-Propyl-N-methylpyrrolidinium bis(fluorosulfonyl)imide $\quad\left(\mathrm{Pyr}_{1,3} \mathrm{FSI}, \quad 99.9 \%\right.$, Solvionic). The ionic liquid was dried under vacuum overnight; the water content as measured by Karl Fischer Titration (C30S KF, Mettler Toledo) was below $15 \mathrm{ppm}$. Electrolyte conductivity was measured using a conductivity meter (Jenway 4510 conductivity meter with a $4 \mathrm{~mm}$ diameter Jenway microvolume conductivity probe) inside a temperature controlled chamber in an argon glove box. The temperature was allowed to stabilise at each temperature point before measurement.
Viscosity was measured using a viscosity meter (Lovis $2000 \mathrm{M}$ microviscometer using a $1.8 \mathrm{~mm}$ diameter capillary and a $1.8 \mathrm{~mm}$ diameter steel ball).

Operando XRD of graphite was performed on a Rigaku Smartlab X-ray diffractometer using a HyPix-3000 2d detector in stationary capture mode. The cell was cycled at a current density of $10 \mathrm{~mA} \mathrm{~g}^{-1}$ and X-ray diffractograms were taken every 10 minutes.

Except where otherwise specified, the electrochemical tests were performed in three electrode cells with potassium metal acting as counter and reference electrode. The surface of the potassium metal was mechanically cleaned before assembly to remove oxides and other contaminants. It was then immersed for $15 \mathrm{~h}$ in the electrolyte. This was necessary because of the high reactivity of the potassium metal. After $15 \mathrm{~h}$ the impedance spectrum of a symmetrical alkali-metal cell stabilizes. On the other hand, the impedance spectra did not stabilize with either the $1 \mathrm{M} \mathrm{KFSI}$ or a $0.7 \mathrm{M} \mathrm{KPF}_{6}$ in EC:DEC (1:1 vol) electrolytes (Supplementary Figure 13). Two-electrode measurements were performed in 2032 coin cell, with potassium metal acting both as counter and reference electrodes. In this configuration the polarization of the reference electrode was calculated to be below $60 \mathrm{mV}$ and $15 \mathrm{mV}$ for the cycle-life tests of the graphite and the KMF electrodes respectively. The full cell was tested in three-electrode configuration with a potassium metal reference electrode.

Density functional theory calculations: Density functional theory (DFT) calculations were performed with the Vienna Ab Initio Simulation Package (VASP). ${ }^{34,35}$ For the fully reduced, monoclinic material $\mathrm{K}_{2} \mathrm{Mn}\left[\mathrm{Fe}(\mathrm{CN})_{6}\right]$, atomic positions were taken from the refinement of the synchrotron XRD from this study. For cubic $\mathrm{KMn}\left[\mathrm{Fe}(\mathrm{CN})_{6}\right]$, initial atomic positions were taken from operando XRD data $^{19}$ and the atomic positions, but not the lattice parameters, were allowed to relax. Spin-polarized calculations were performed with the parameterized Perdew-Burke-Ernzerhof exchange-correlation function. ${ }^{36}$ To account for the over-delocalization of electrons in d orbitals on the metal centers, the $\mathrm{DFT}+\mathrm{U}$ method was used. Values of the Hubbard $\mathrm{U}$ parameters of $5.0 \mathrm{eV}$ for manganese and $4.3 \mathrm{eV}$ for iron were taken from literature. ${ }^{37}$ The wavefunction cutoffs were $400 \mathrm{eV}$. The maximum energy difference for convergence during electronic convergence was $10-4 \mathrm{eV}$. The maximum sum of the forces on all atoms for convergence during ionic convergence was $0.01 \mathrm{eV}^{-1}$. 


\section{Acknowledgements}

This publication arises from research funded by the John Fell Oxford University Press Research Fund. The authors would like to acknowledge the financial support of the Henry Royce Institute (through UK Engineering and Physical Science Research Council grant EP/R010145/1) for capital equipment. M.F. and R.R. acknowledge the Italian Ministry of University and Research (MIUR) for the grant D̀ipartimenti di Eccellenza- 2017 Materials For Energy! IC acknowledges support from the Modentech studentship. We are grateful to the David Cockayne Center for Electron Microscopy for the use of their electron microscopes. The authors would like to acknowledge the use of the University of Oxford Advanced Research Computing (ARC) facility in carrying out this work. We thank Diamond Light Source (I11 Beamline) for the provision of beamtime via the Block award grant and I11 beamline staff for the assistance with data collection. We would like to acknowledge Dr. Emory Chan for advice in development of the high-throughput workflow. Work at the Molecular Foundry was supported by the Office of Science, Office of Basic Energy Sciences, of the U.S. Department of Energy under Contract No. DE-AC02-05CH11231.

\section{Author contributions}

M.F., S.W., and M.P. conceived and designed the experiments. M.F., S.W. K.H. performed the experiments, analyzed the data and wrote the manuscript with inputs from all the authors. I.C. assisted in the fabrication of electrodes, build and test full cells and provided expertise regarding electrochemical testing. J.F. prepared the ionic liquid electrolyte and tested its physico-chemical properties. R.R. provided experimental advice. The project was supervised by M.P.

\section{Competing Interests}

The authors declare no competing interests.

\section{References}

1. Vaalma, C., Buchholz, D., Weil, M. \& Passerini, S. A cost and resource analysis of sodium-ion batteries. Nature Reviews Materials 3 (2018).

2. Olivetti, E. A., Ceder, G., Gaustad, G. G. \& Fu, X. Lithium-Ion Battery Supply Chain Considerations: Analysis of Potential Bottlenecks in Critical Metals. Joule 1, 229-243 (2017).
3. Yabuuchi, N., Kubota, K., Dahbi, M. \& Komaba, S. Research development on potassium-ion batteries. Chemical Reviews (2020).

4. Chen, L. et al. Readiness Level of SodiumIon Battery Technology: A Materials Review. Advanced Sustainable Systems 2 (2018).

5. Okamoto, Y. Density functional theory calculations of alkali metal ( $\mathrm{Li}, \mathrm{Na}$, and $\mathrm{K}$ ) graphite intercalation compounds. Journal of Physical Chemistry C 118, 16-19 (2014).

6. Luo, W. et al. Potassium Ion Batteries with Graphitic Materials. Nano Letters 15, 76717677 (2015).

7. Komaba, S., Hasegawa, T., Dahbi, M. \& Kubota, K. Potassium intercalation into graphite to realize high-voltage/high-power potassiumion batteries and potassium-ion capacitors. Electrochemistry Communications 60, 172-175 (2015).

8. Zhao, J., Zou, X., Zhu, Y., Xu, Y. \& Wang, C. Electrochemical Intercalation of Potassium into Graphite. Advanced Functional Materials 26, 8103-8110 (2016).

9. Deng, T. et al. Layered P2-Type $\mathrm{K} 0.65 \mathrm{Fe} 0.5 \mathrm{Mn} 0.5 \mathrm{O} 2$ Microspheres as $\mathrm{Su}-$ perior Cathode for High-Energy Potassium-Ion Batteries. Advanced Functional Materials 28, 1-9 (2018).

10. Kim, H. et al. A New Strategy for High-Voltage Cathodes for K-Ion Batteries: Stoichiometric KVPO4F. Advanced Energy Materials 8, 1-12 (2018).

11. Lin, X., Huang, J., Tan, H., Huang, J. \& Zhang, B. K3V2(PO4)2F3 as a robust cathode for potassium-ion batteries. Energy Storage Materials 16, 97-101 (2019).

12. Hurlbutt, K., Wheeler, S., Capone, I. \& Pasta, M. Prussian Blue Analogs as Battery Materials. Joule 2, 1950-1960 (2018).

13. Scholz, F. \& Dostal, A. The Formal Potentials of Solid Metal Hexacyanometalates. Angewandte Chemie International Edition in English 34, 2685-2687 (1995).

14. Wu, X., Jian, Z., Li, Z. \& Ji, X. Prussian white analogues as promising cathode for nonaqueous potassium-ion batteries. Electrochemistry Communications 77, 54-57 (2017).

15. He, G. \& Nazar, L. F. Crystallite Size Control of Prussian White Analogues for Nonaqueous Potassium-Ion Batteries. ACS Energy Letters 2, 1122-1127 (2017). 
16. Huang, B. et al. Prussian Blue $[\mathrm{K} 2 \mathrm{FeFe}(\mathrm{CN}) 6]$ Doped with Nickel as a Superior Cathode: An Efficient Strategy to Enhance Potassium Storage Performance. ACS Sustainable Chemistry and Engineering 7, 16659-16667 (2019).

17. Xue, L. et al. Low-Cost High-Energy Potassium Cathode. Journal of the American Chemical Society 139, 2164-2167 (2017).

18. Jiang, L. et al. Building aqueous K-ion batteries for energy storage. Nature Energy 4, 495-503 (2019).

19. Bie, X., Kubota, K., Hosaka, T., Chihara, K. \& Komaba, S. A novel K-ion battery: hexacyanoferrate(II)/graphite cell. J. Mater. Chem. A 5, 4325-4330 (2017).

20. Hosaka, T., Kubota, K., Kojima, H. \& Komaba, S. Highly concentrated electrolyte solutions for $4 \mathrm{~V}$ class potassium-ion batteries. Chemical Communications 54, 8387-8390 (2018).

21. Fan, L., Ma, R., Zhang, Q., Jia, X. \& Lu, B. Graphite Anode for a Potassium-Ion Battery with Unprecedented Performance. Angewandte Chemie - International Edition 410082, 1050010505 (2019).

22. Kubota, K., Dahbi, M., Hosaka, T., Kumakura, S. \& Komaba, S. Towards K-ion and Na-ion Batteries as Beyond Li-ion. Chemical Record 18, 459-479 (2018).

23. Dahn, J. R., Burns, J. C. \& Stevens, D. A. Importance of coulombic efficiency measurements in $\mathrm{R} \& \mathrm{D}$ efforts to obtain long-lived li-ion batteries. Electrochemical Society Interface $\mathbf{2 5}$, 75-78 (2016).

24. Chen, R. et al. A chemical inhibition method to synthetize highly crystalline Prussian blue analogs for sodium-ion battery cathodes. ACS Applied Materials $\&$ Interfaces acsami.6b10884 (2016).

25. Wu, X. et al. Highly Crystallized $\mathrm{Na} 2 \mathrm{CoFe}(\mathrm{CN}) 6$ with Suppressed Lattice Defects as Superior Cathode Material for SodiumIon Batteries. ACS Applied Materials and Interfaces 8, 5393-5399 (2016).

26. Moritomo, Y., Kurihara, Y., Matsuda, T. \& Kim, J. Structural phase diagram of Mn-Fe cyanide against cation concentration. Journal of the Physical Society of Japan 80, 103601 (2011).

27. Zhou, A. et al. Size-, Water-, and DefectRegulated Potassium Manganese Hexacyanoferrate with Superior Cycling Stability and
Rate Capability for Low-Cost Sodium-Ion Batteries. Small 1902420 (2019).

28. Jiang, X., Zhang, T., Yang, L., Li, G. \& Lee, J. Y. A Fe/Mn-Based Prussian Blue Analogue as a K-Rich Cathode Material for PotassiumIon Batteries. ChemElectroChem 4, 2237-2242 (2017).

29. Fiore, M. et al. Electrochemical characterization of highly abundant, low cost iron (III) oxide as anode material for sodium-ion rechargeable batteries. Electrochimica Acta 269, 367377 (2018).

30. Song, J. et al. Removal of interstitial $\mathrm{H} 2 \mathrm{O}$ in hexacyanometallates for a superior cathode of a sodium-ion battery. Journal of the American Chemical Society 137, 2658-2664 (2015).

31. Liu, J. et al. Unraveling the Potassium Storage Mechanism in Graphite Foam. Advanced Energy Materials 1900579, 1-11 (2019).

32. Jian, Z., Luo, W. \& Ji, X. Carbon Electrodes for K-Ion Batteries. Journal of the American Chemical Society 137, 11566-11569 (2015).

33. Carboni, M., Naylor, A. J., Valvo, M. \& Younesi, R. Unlocking high capacities of graphite anodes for potassium-ion batteries. RSC Advances 9, 21070-21074 (2019).

34. Kresse, G. \& Hafner, J. Ab initio molecular dynamics for liquid metals. Physical Review $B$ 47, 558-561 (1993).

35. Kresse, G. \& Hafner, J. Ab initio molecular-dynamics simulation of the liquidmetalamorphous- semiconductor transition in germanium. Physical Review B 49, 1425114269 (1994).

36. Kresse, G. \& Furthmüller, J. Efficient iterative schemes for ab initio total-energy calculations using a plane-wave basis set. Physical Review $B$ - Condensed Matter and Materials Physics 54, 11169-11186 (1996).

37. Xiao, P., Song, J., Wang, L., Goodenough, J. B. \& Henkelman, G. Theoretical study of the structural evolution of a $\mathrm{Na} 2 \mathrm{FeMn}(\mathrm{CN}) 6$ cathode upon $\mathrm{Na}$ intercalation. Chemistry of Materials 27, 3763-3768 (2015). 